\title{
ВАРИАТИВНЫЙ УЧЕТ ЦЕНООБРАЗУЮЩИХ ФАКТОРОВ ПРИ КАДАСТРОВОЙ ОЦЕНКЕ ЗЕМЕЛЬ (НА ПРИМЕРЕ ГОРОДА САНКТ-ПЕТЕРБУРГ)
}

\author{
Лепихина Ольга Юрьевна', \\ Olgalepikhina1984@gmail.com \\ Правдина Екатерина Александровна', \\ eanes@yandex.ru \\ ' Санкт-Петербургский горный университет, \\ Россия, 199026, г. Санкт-Петербург, 21-я линия В.О., 2.
}

Актуальность исследования. Земля является важнейшим ресурсом, значимость которого в настоящее время очень сложно переоценить. Будучи средством производства, в то же время она выступает в качестве пространственного базиса для жизнедеятельности населения. В современных условиях особенно остро стоит вопрос обеспечения рационального и эффективного использования земель. Для этой цели необходим комплексный анализ и оценка ее экономических, климатических, экологических, геологических и иных характеристик. Одной из разновидностей такой оценки является определение кадастровой стоимости земель. В нашей стране она используется, в первую очередь, для налогообложения, в связи с чем важным становится вопрос о необходимости получения точных и объективных результатов оценки. Современные методики не совершенны, и их применение не всегда обеспечивает корректные и справедливые стоимости земель. Так, к примеру, в них недостаточно внимания уделяется обоснованию исходных ценообразующих факторов, а также корректному выбору их учета при кадастровой оценке. Влияние каждого ценообразующего фактора можно выразить с помощью оценочных показателей разной размерности. Настоящая статья посвящена анализу зависимости кадастровой стоимости земель от выбранного варианта учета факторов на примере земельных участков, расположенных на Васильевском острове города Санкт-Петербурга.

Цель: исследование влияния вариативного учета ценообразующих факторов на итоговую стоимость земель населенных пунктов для дальнейшего уточнения и совершенствования методического обеспечения кадастровой оценки земель.

Методы. Анализ района местоположения объектов исследования выполнен на основе изучения картографических источников, а также путем визуального осмотра объектов недвижимости. Сбор значений оценочных показателей кадастровой стоимости земельных участков осуществлялся методами пространственного анализа и моделирования данных в геоинформационной системe Maplnfo. Выборки рыночных данных о земельных участках производились классическими методами статистического сбора и обработки данных с применением программных приложений пакета MS Office и SPSS. Для построения статистических моделей кадастровой стоимости земель использован корреляционно-регрессионный метод. В целях определения взаимозависимых оценочных показателей при трех рассматриваемых вариантах использован метод “корреляционных плеяд» П.В. Терентьева. Результаты. При проведении кадастровой оценки земель необходимо принимать во внимание наличие вариативности учета факторов с помощью разноразмерных оценочных показателей. Выбор того или иного показателя, характеризующего влияние фактора, должен быть обоснован. Корреляционный анализ показателей выявил разную степень их взаимозависимости, что свидетельствует о том, что они не всегда могут быть взаимозаменяемы при построении статистических моделей. Установлено, что от выбора состава исходных оценочных показателей факторов зависит их структура, состав наиболее значимых показателей, а также рассчитанные кадастровые стоимости земельных участков.

\section{Ключевые слова:}

Земельный участок, природный ресурс, кадастровая оценка, ценообразующий фактор,

оценочный показатель, корреляционно-регрессионный метод, метод корреляционных плеяд.

\section{Введение}

Наша страна обладает ценнейшими природными ресурсами, центральное место среди которых занимает земля. Несмотря на обширность распространения по площади, данный ресурс ограничен, в связи с чем актуальной во все времена остается проблема обеспечения рационального его использования. Земля обладает уникальными природными характеристиками, исследование которых необходимо для обоснования наиболее эффективного ее использования в хозяйственной деятельности человека.

Однако землю нужно рассматривать не только как природный ресурс, но и как объект социальноэкономических отношений, обладающий определенной оценочной стоимостью.

Одной из разновидностей оценки земель в нашей стране является определение ее кадастровой стоимости. Помимо обозначенных ранее целей, связанных с обеспечением рационального и эффективного использования земель, указанная оценка используется также для налогообложения.

Тема кадастровой оценки земель и особенно ее методического обеспечения нашла отражение во многих публикациях отечественных исследователей. Так, например, в работах Ю.В. Чернецкой [1], Ю.И. Шабаевой [2], О.А. Трибуц [3] проанализированы основные факторы, оказывающие влияние на стоимость земель в населенных пунктах. Исследования $[4,5]$ посвящены экспертному подходу к массовой оценке. Работы Т.И. Балтыжаковой $[6,7]$, П.М. Демидовой и А.М. Рыбкиной [8] освещают особенности применения статистических методов расчета кадастровой стоимости земель. В статьях А.В. Чирухина [9, 10] содержатся требования, предъявляемые к рыночной информации, используемой для проведения кадастровой оценки. 
За рубежом тема кадастровой оценки также весьма актуальна. Основные вопросы, волнующие представителей западного научного сообщества, связаны с точностью формируемых статистических моделей массовой оценки земель [11], методами определения значений ценообразующих факторов [12], анализом специфики и возможностей применения массовой и индивидуальной оценки объектов для условий сложившегося рынка недвижимости $[13,14]$.

При государственной кадастровой оценке возможно возникновение разного рода ошибок и нарушений, обусловливающих необъективную итоговую величину кадастровой стоимости. $К$ сожалению, это приводит к общественной неудовлетворенности результатами оценки и обусловливает возрастающее количество обращений об оспаривании стоимости в суд $[15,16]$. Отмеченная ситуация возникает, в том числе, из-за несовершенства методического обеспечения оценочных работ [17-19].

Несмотря на утвержденный единый подход массовой оценки, содержание этапов метода жестко не регламентируются. Так, в части процедуры проведения оценки, связанной с обоснованием исходных ценообразующих факторов, отсутствует обязательный для учета перечень данных факторов, а также метод их учета.

Известно, что на стоимость земель населенных пунктов главным образом оказывают влияние следующие факторы: местоположение, ландшафтнорекреационной характеристики, уровень развития транспортной и социальной инфраструктуры, инженерное благоустройство территории, экология и другие $[20,21]$. В настоящей статье предлагается исследовать зависимость кадастровой стоимости земель от ряда ценообразующих факторов с учетом возможного их вариативного учета при оценке. Авторами выдвигается гипотеза о том, что выбор того или иного варианта учета фактора с помощью разноразмерных показателей окажет влияние на структуру итоговой статистической модели и, следовательно, на рассчитанные стоимости земельных участков.

Настоящая статья является продолжением научных исследований авторов, посвященных особенностям учета ценообразующих факторов при кадастровой оценке земель различных по типу населенных пунктов России [22].

\section{Методы и предмет исследования}

Предметом исследования в настоящей работе являются факторы, показатели и кадастровая стоимость земельных участков, предназначенных для размещения домов многоэтажной жилой застройки, расположенных в Василеостровском районе города Санкт-Петербург.

Общей теоретической и методологической основой работы явился комплексный подход, включающий анализ нормативно-правовой и научной литературы в области кадастровой оценки земель населенных пунктов. Для разработки и обоснования положений работы применялись современные экономико-математические и статистические методы: системный анализ, сравнительный анализ, корреляционно-регрессионный анализ, метод корреляционных плеяд. Решение поставленных задач осуществлялось посредством использования современных технических средств и программных продуктов (MapInfo Pro 15.0, Microsoft Excel, SPSS Statistics 22).

\section{Результаты исследования}

В качестве объектов исследования в Василеостровском районе города Санкт-Петербург было выделено сто тестовых участков указанного ранее вида использования.

Анализ влияния разноразмерных ценообразующих факторов на кадастровую стоимость земель осуществлен на примере факторов, отражающих доступность следующих объектов социальной инфраструктуры:

1) школы;

2) детские дошкольные упреждения (далее ДДУ);

3) высшие учебные заведения (далее - вузы) и средние специальные учебные учреждения (далее ССУЗы);

4) учреждения культуры;

5) объекты медицины;

6) аптеки;

7) объекты индустрии отдыха и развлечений;

8) организации сферы обслуживания;

9) продуктовые магазины;

10)торговые комплексы.

В рамках настоящего исследования предлагается выразить отмеченные выше ценообразующие факторы через систему оценочных показателей разной размерности. Для проверки гипотезы исследования на основании проведенного анализа факторов социальной инфраструктуры выбраны три варианта их учета при кадастровой оценке (табл. 1).

Первый вариант учета фактора социальной инфраструктуры (измерение расстояния от оцениваемого объекта до объекта социальной инфраструктуры) предлагается Методикой государственной кадастровой оценки земель населенных пунктов 2007 г. и заключается в определении значений показателей путем измерения расстояния от оцениваемого объекта до объекта фактора. При этом расстояния измеряются от середины каждого оцениваемого объекта до середины объекта фактора по границам дорог, земельных участков, водоемов.

Второй вариант учета фактора (определение площади оцениваемого объекта, попадающей в зону доступности (влияния) объекта инфраструктуры) предложен в рамках Методики государственной кадастровой оценки земель поселений 2002 г. Он заключается в построении радиусов пешеходной доступности для каждого объекта социальной инфраструктуры и измерении площади оцениваемого объекта, попавшей в данный радиус. 
Таблица 1. Факторы социальной инфраструктуры и варианты их учета с помощью трех разноразмерных оценочных показателей с указанием типа статистической шкалы

Table 1. $\quad$ Factors of social infrastructure and options for their accounting using three different-sized indicators with their statistical scales

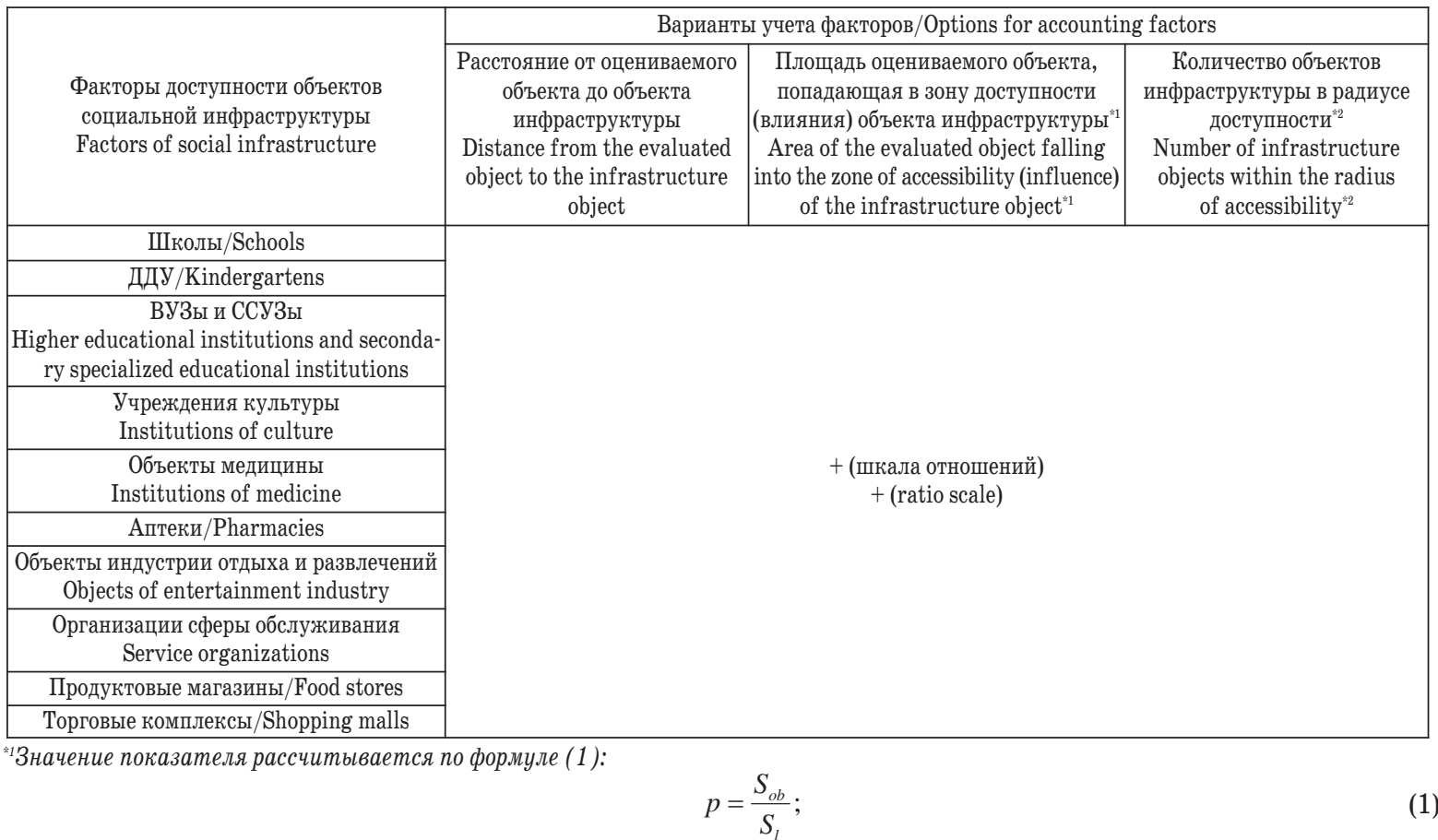

$S_{\text {оb }}$ - площадь, попадающая в зону влияния объекта инфраструктуры; $S_{l}$ - общая площадь земельного участка.

${ }^{* 2}$ В соответствии с СП 42.13330.2011 Градостроительство. Планировка и застройка городских и сельских поселений.

${ }^{*}$ Indicators are calculated by the formula (1). $S_{o b}$ - the area is the evaluated object falling into the zone of accessibility; $S_{l}$ is land parcel area.

${ }^{* 2}$ In accordance with SP 42.13330.2011 Urban planning. Planning and development of urban and rural settlements

Таблица 2. Коэффиииенты корреляиии для трех методов учета факторов

Table 2. Correlation coefficients for three options of accounting the factors

\begin{tabular}{|c|c|c|c|c|c|c|c|c|c|c|c|}
\hline 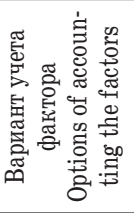 & 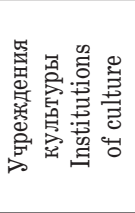 & 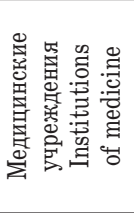 & 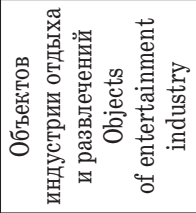 & 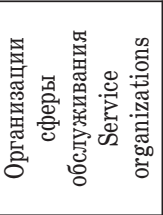 & 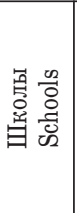 & 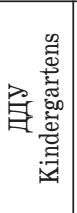 & 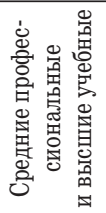 & 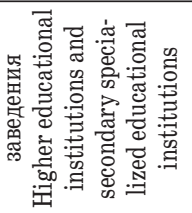 & 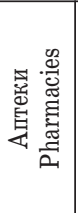 & 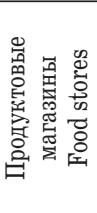 & 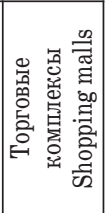 \\
\hline 1 & 0,28 & 0,09 & $-0,38$ & 0,24 & $-0,02$ & 0,07 & & $-0,26$ & 0,15 & 0,10 & 0,10 \\
\hline 2 & $-0,38$ & 0,29 & $-0,11$ & $-0,11$ & 0,44 & 0,24 & & 0,19 & $-0,19$ & 0,17 & 0,15 \\
\hline 3 & $-0,05$ & $-0,08$ & $-0,05$ & $-0,04$ & $-0,08$ & $-0,05$ & & $-0,05$ & $-0,05$ & $-0,04$ & $-0,05$ \\
\hline
\end{tabular}

Также в рамках данного исследования предлагается еще один метод учета фактора, а именно, подсчет количества объектов инфраструктуры в радиусе пешеходной доступности. Как и во втором варианте, строятся радиусы доступности для каждого отдельного объекта, и считается количество попаданий оцениваемого объекта в радиусы доступности объектов инфраструктуры.

Таким образом, показана вариативность учета ценообразующих факторов с помощью разноразмерных показателей.

В геоинформационной системе MapInfo Pro 15.0 был создан графический проект, содержащий следующие сведения: местоположение и границы земельных участков, данные об объектах социальной инфраструктуры. Средствами пространствен- ного анализа и моделирования данных определены значения показателей факторов для трех рассматриваемых вариантов их учета (рис. 1).

По результатам измерений были созданы три группы показателей ценообразующих факторов социальной инфраструктуры. В первой группе показатели, полученные методом измерения расстояния от оцениваемого объекта до объекта показателя, во второй группе - показатели, полученные методом подсчета количества объектов показателя в радиусе доступности, в третьей группе показатели, полученные методом измерение площади оцениваемого объекта, попадающей в зону доступности (влияния) объекта показателя.

Информация о рыночной стоимости земельных участков района исследования собрана из следую- 
щих источников: баз рыночных стоимостей объектов недвижимости Василеостровского района, приложений к отчету об определении кадастровой стоимости в Санкт-Петербурге, данных портала Росреестра.

Для определения влияния ценообразующих факторов на стоимость земельных участков в каждом из трех вариантов учета были рассчитаны коэффициенты корреляции между значениями рыночных стоимостей земельных участков и каждым из показателей (табл. 2).

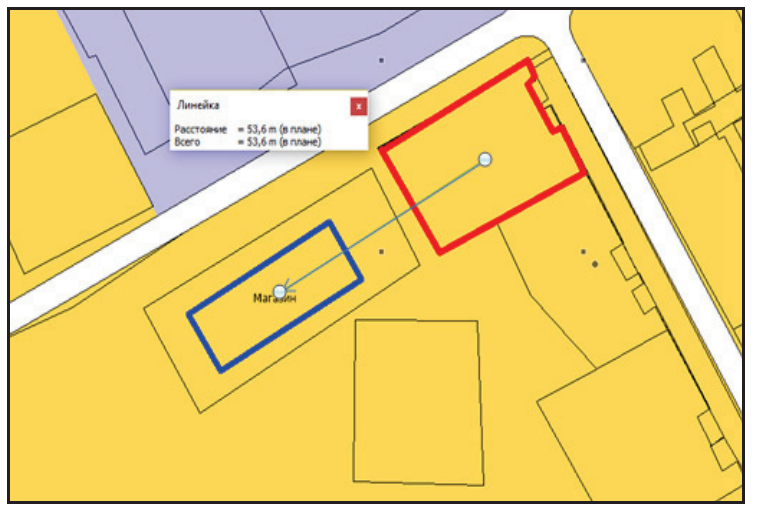

$a / a$

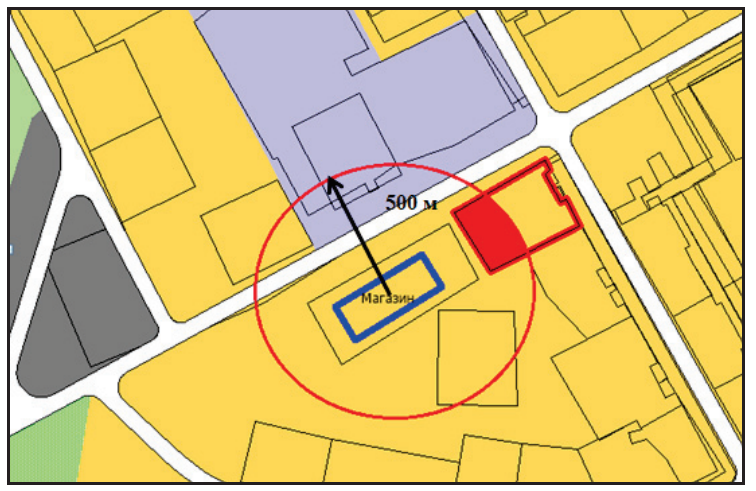

$\sigma / b$

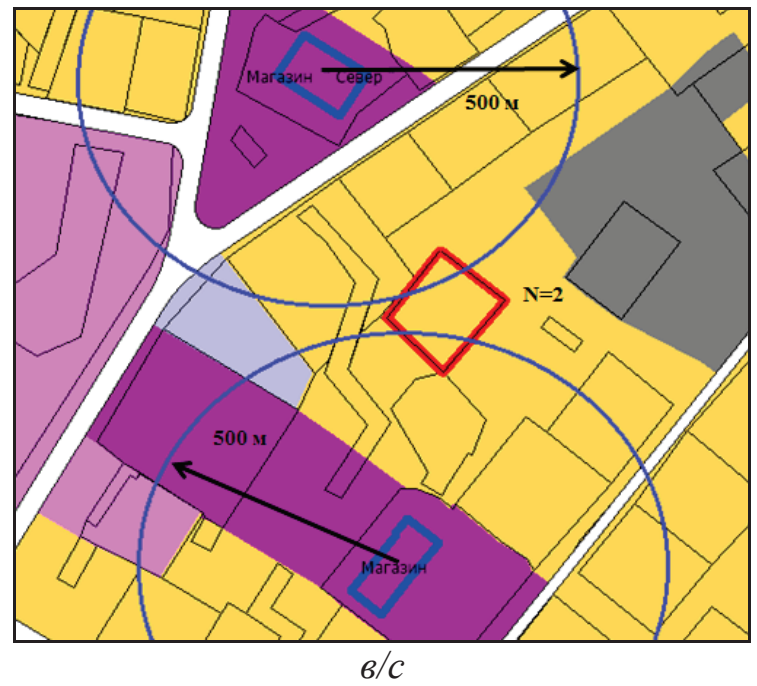

Pис.1. Определение значений оценочных показателей по первому (а), второму (б) и третьему (в) варианту

Fig. 1. Determination of estimated indicators by the first (a), second (b) and third (c) option
По полученным значениям можно сделать вывод о том, что при разных вариантах учета факторов степень взаимосвязи их с рыночными стоимостями земель неодинакова, в связи с чем они не могут быть взаимозаменяемы при кадастровой оценке земель.

Нужно отметить, что наличие устойчивого перечня ценообразующих факторов кадастровой стоимости земель позволяет сократить общее количество учитываемых факторов путем выявления наиболее значимых, содержащих наибольшую информацию о значениях остальных. С целью выявления зависимостей между отдельными факторами был использован метод «корреляционных плеяд» П.В. Терентьева.

Данный метод предназначен для кластер-анализа признаков и заключается в построении графической структуры - «корреляционных плеяд». Корреляционные плеяды - это способ отображения информации о корреляциях, который помогает их структурировать, проводить объединение коррелирующих факторов. Корреляционная плеяда - это граф с множеством вершин, состоящий из узлов (факторов) и ребер (корреляционных связей, по величине превосходящих назначаемое пороговое значение коэффициента корреляции). «Ядром» каждой плеяды выбирают узел (фактор), имеющий максимальное количество ребер. Совокупность «ядер» корреляционных плеяд соответствует перечню наиболее значимых факторов.

Для проверки полученных перечней показателей на мультиколлинеарность с помощью программы SPSS Statistics 22 была получена матричная таблица корреляций каждого отдельного фактора друг с другом в пределах метода. Наличие больших по модулю (выше 0,7-0,8) значений коэффициентов парной корреляции свидетельствует о возможных проблемах с качеством получаемых оценок. В расчетах было принято исключить те факторы, значение коэффициентов которых более 0,75. Если факторы модели коллинеарны, то они дублируют друг друга и один из них рекомендуется исключить из анализа. Предпочтение отдается не фактору, более сильно связанному с результатом, а фактору, который при наибольшей связи с результатом имеет наименьшую тесноту связи с другими факторами.

По результатам расчетов для первого и третьего вариантов учета был исключен фактор «Учреждения культуры». Для второго варианта учета был исключен фактор «Организации сферы обслуживания» .

Также были получены уровни статистической значимости $(p)$. Эти уровни могут иметь различное числовое выражение в интервале от 0 до 1. При $p<=0,05$, результаты считаются статистически достоверными (значимыми), при $p<=0,01$ результаты получены на высоком уровне статистической значимости. Таким образом, для дальнейшего построения схем корреляционных плеяд отбираются те коэффициенты корреляции между факторами, 
показатели статистической значимости которых принимают значения $p<=0,05(*)$ или $p<=0,01(* *)$.

На схемах корреляционных плеяд приняты следующие обозначения:

- обратная связь между факторами обозначается пунктиром;

- корреляция на уровне статистической значимости 0,01 обозначается одинарной линией;

- корреляция на уровне статистической значимости 0,05 обозначается двойной линией.

Схемы корреляционных плеяд, полученные для трех вариантов учета ценообразующих факторов, представлены на рис. 2.

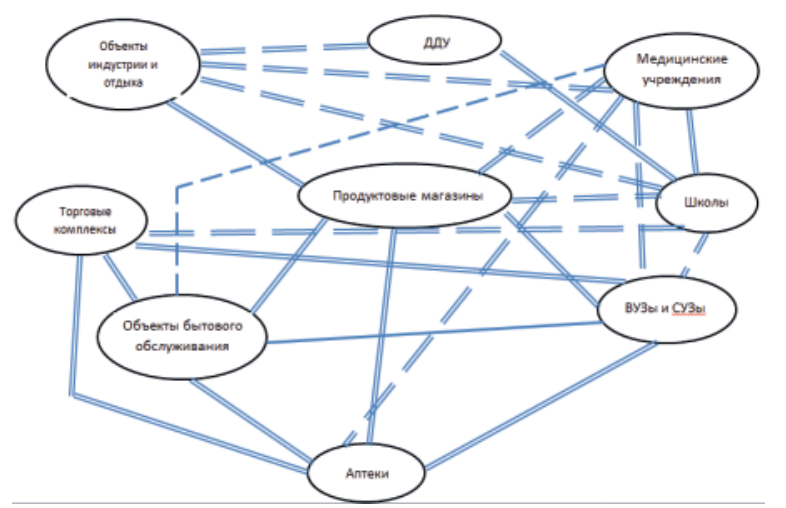

$a / a$
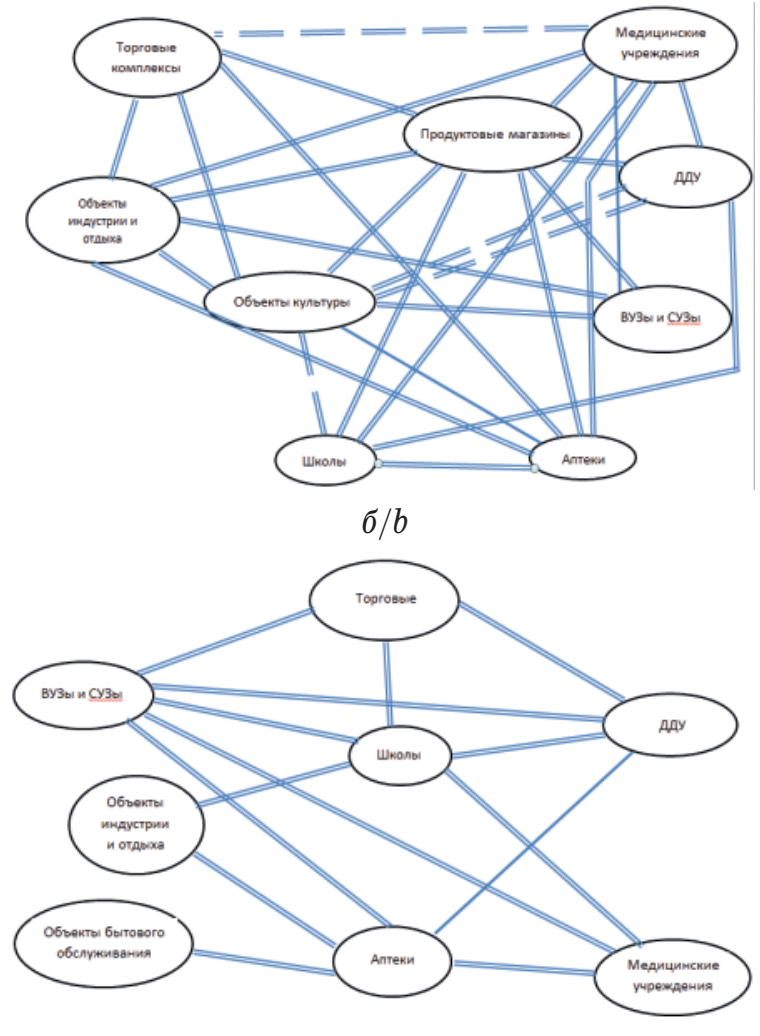

$8 / c$

Pис. 2. Схема корреляиионных плеяд для первого (а), второго (б) и третьего (в) варианта

Fig. 2. Correlation galaxies for the first (a), second (b) and third (c) option
Схемы корреляционных плеяд визуально демонстрируют различие в зависимостях между показателями при разных вариантах учета ценообразующих факторов. Наибольшее количество зависимостей наблюдается на схеме, построенной по результатам второго метода, и можно сделать вывод о том, что в данном случае возможно сократить количество факторов, оказывающих влияние на итоговую стоимость земельного участка, что упростит структуру статистической модели при расчете кадастровой стоимости земли.

Для дальнейшего доказательства того факта, что выбор того или иного варианта учета факторов окажет влияние на вид итоговой статистической модели и рассчитанные стоимости объектов недвижимости, построим модель кадастровой стоимости для трех вариантов учета этих факторов.

Так как действующие методики кадастровой оценки строго не регламентируют перечень факторов стоимости, отбор наиболее значимых ценообразующих факторов производится на усмотрение оценщика. В данном случае в качестве факторов стоимости для исследуемого вида разрешенного использования выделим в первую очередь рассмотренные выше факторы социальной инфраструктуры, а также дополнительные факторы, учитываемые в современной практике оценки: площадь земельного участка, развитие транспортной инфраструктуры, рекреационная ценность территории.

Построение статистических моделей расчета кадастровой стоимости земельных участков осуществляется в следующей последовательности:

1) выбор факторов стоимости для построения моделей;

2) построение моделей расчета кадастровой стои-

мости земельных участков;

3) анализ качества статистических моделей.

Для отбора ценообразующих факторов следует определить коэффициенты значимости факторов по формуле (2):

$$
R_{K}=\frac{r_{k y}}{r_{\max }},
$$

где $r_{k y}$ - коэффициенты частной корреляции; $r_{\max }-$ максимальный из найденных коэффициентов частной корреляции.

Результаты расчетов для трех рассматриваемых вариантов представлены в табл. 3 .

Цель построения моделей расчета кадастровой стоимости земельных участков (модель расчета) состоит в получении статистически значимой и качественной модели расчета.

Под моделью расчета понимается математическая формула, отображающая связь между зависимой переменной и значениями соответствующих факторов стоимости. В качестве зависимой переменной выступает удельный показатель кадастровой стоимости (УПКС).

Для расчета УПКС действующим методическим обеспечением кадастровой оценки земель рассматриваемого вида разрешенного использования регламентирован метод регрессионной модели. 
Таблица 3. Выбор факторов стоимости для трех вариантов учета на основе анализа значимости коэффициентов корреляции

Table 3. $\quad$ Choice of factors for three options by analysis of the correlation coefficients statistical importance

\begin{tabular}{|c|c|c|c|c|c|c|c|c|c|c|c|c|c|}
\hline Фактор/Factor & $\Phi 1 / \mathrm{F} 1$ & $\Phi 2 / \mathrm{F} 2$ & Ф3/F3 & $\Phi 4 / \mathrm{F} 4$ & $\Phi 5 / \mathrm{F} 5$ & $\Phi 6 / \mathrm{F} 6$ & $\Phi 7 / F 7$ & $\Phi 8 / \mathrm{F} 8$ & $\Phi 9 / \mathrm{F} 9$ & $\Phi 10 / \mathrm{F} 10$ & $\Phi 11 / \mathrm{F} 11$ & $\Phi 12 / \mathrm{F} 12$ & $\Phi 13 / \mathrm{F} 13$ \\
\hline \multicolumn{14}{|c|}{ Первый вариант учета/First option of accounting } \\
\hline$r_{k y}$ & $-0,1$ & 0,0 & 0,3 & 0,1 & $-0,4$ & 0,2 & $-0,0$ & 0,1 & $-0,3$ & 0,2 & 0,1 & 0,1 & 0,8 \\
\hline$R_{k}$ & $-0,1$ & 0,0 & 0,4 & 0,1 & $-0,5$ & 0,3 & $-0,0$ & 0,1 & $-0,4$ & 0,2 & 0,1 & 0,1 & 1 \\
\hline $\begin{array}{l}\text { Заключение о значимости } \\
\text { Conclusion on significance }\end{array}$ & нет $/$ no & нет $/$ по & да/yes & нет/nо & да/yes & да/yes & нет/no & нет/nо & да/yes & нет/no & нет $/$ nо & нет/no & да/yes \\
\hline \multicolumn{14}{|c|}{ Второй вариант учета/Second option of accounting } \\
\hline$r_{k y}$ & $-0,3$ & 0,4 & $-0,4$ & 0,3 & $-0,1$ & $-0,1$ & 0,4 & 0,2 & 0,2 & $-0,2$ & 0,2 & 0,2 & 0,8 \\
\hline$R_{k}$ & $-0,4$ & 0,5 & $-0,5$ & 0,4 & $-0,1$ & $-0,1$ & 0,6 & 0,3 & 0,2 & $-0,2$ & 0,2 & 0,2 & 1 \\
\hline $\begin{array}{l}\text { Заключение о значимости } \\
\text { Conclusion on significance }\end{array}$ & да/yes & да/yes & да/yes & да/yes & нет $/$ no & нет/no & да/yes & да/yes & нет/no & нет $/$ по & нет/no & нет $/$ nо & да/yes \\
\hline \multicolumn{14}{|c|}{ Третий вариант учета/Third option of accounting } \\
\hline$r_{k y}$ & $-0,1$ & $-0,0$ & $-0,1$ & $-0,1$ & $-0,1$ & $-0,0$ & $-0,1$ & $-0,1$ & $-0,1$ & $-0,1$ & $-0,0$ & $-0,1$ & 0,8 \\
\hline$R_{k}$ & $-0,1$ & $-0,0$ & $-0,1$ & $-0,1$ & $-0,1$ & $-0,1$ & $-0,1$ & $-0,1$ & $-0,1$ & $-0,1$ & $-0,1$ & $-0,1$ & 1 \\
\hline $\begin{array}{l}\text { Заключение о значимости } \\
\text { Conclusion on significance }\end{array}$ & \multicolumn{12}{|c|}{ нет/no } & да/yes \\
\hline
\end{tabular}

Ф1 - развитие транспортной инфраструктуры; Ф2 - рекреационная ценность территории; Ф3-учреждения культуры; Ф4-ледииинские учреждения; $\Phi 5$ - объекты индустрии отдыха и развлечений; Ф6 - организации сферы обслуживания; вузы и ССУЗы; Ф10 - аптеки; Ф11 - продуктовые магазины; $Ф 12$ - торговые колплексы; Ф13 - площадь участка.

F1 - development of transport infrastructure; F2 - recreational value of the territory; F3-institutions of culture; F4 - institutions of medicine; F5 - objects of entertainment industry; F6 - service organizations; F7 - schools; F8 - kindergartens; F9 - higher educational institutions and secondary specialized educational institutions; F10 - pharmacies; F11-food stores; F12-shopping malls; F13 - land parcel area.

Для построения модели необходима исходная рыночная информация о земельных участках, объем которой должен превосходить количество факторов стоимости более чем в шесть раз.

Таким образом, необходимо иметь рыночную информацию в следующем объеме:

- для первого варианта учета факторов - не менее чем о 36 рыночных сделках;

- для первого варианта учета факторов - не менее чем о 48 рыночных сделках;

- для первого варианта учета факторов - не менее чем о 12 рыночных сделках.

Для построения моделей воспользуемся методами регрессионной статистики.

Уравнение линейной (аддитивной) модели имеет вид (3):

$$
Y=a_{0}+a_{1} \cdot x_{1}+\ldots+a_{n} \cdot x_{n},
$$

где $Y$ - зависимая переменная (рыночная стоимость $1 \mathrm{м}^{2}$ земли или стоимость земельных участков); $x_{1}, \ldots, x_{n}-$ факторы стоимости земельных участков; $a_{0}, a_{1} \ldots a_{n}-$ коэффициенты модели.

Для первого варианта учета факторов регрессионный анализ дал следующие результаты: $R$ квадрат равен 0,74- высокая точность аппроксимации; $p$-значение $<0,05$ - модель значима; значение свободного члена ( $Y$-пересечение) 25426,088.

Уравнение для расчета кадастровой стоимости имеет вид (4):

$$
\begin{gathered}
Y=25426,09-0,29 \cdot x_{1}+4,55 \cdot x_{2}+ \\
+23,24 \cdot x_{3}+7,82 \cdot x_{4}-12,72 \cdot x_{5},
\end{gathered}
$$

где $x_{1}, \ldots, x_{5}-$ значения факторов стоимости земельных участков;

Для третьего варианта: $R$-квадрат равен 0,74 - средняя точность аппроксимации; получен- ное $p$-значение $<0,05$ - модель значима; значение свободного члена (Y-пересечение) $31205,2156$.

Уравнение для расчета кадастровой стоимости имеет вид (5):

$$
Y=31205,2-0,42 \cdot x_{1},
$$

где $x_{1}$ - значение фактора стоимости земельных участков.

Модель, полученная по второлу варианту учета факторов, оказалась незначима, в связи с чем в дальнейшем данный вариант рассмотрен не был.

Также для проверки статистической значимости модели используется критерий Фишера (6):

$$
F=\frac{(N-m-1) R^{2}}{m\left(1-R^{2}\right)},
$$

где $N$ - количество объектов в выборке; $m$ - количество факторов стоимости, используемых в построении модели; $R^{2}$ - коэффициент детерминации построенной модели.

Модель считается статистически значимой, если найденное значение $F$-критерия превышает пороговое значение $F_{\text {табл }}$ при заданном уровне значимости 0,05 .

Результаты расчетов приведены в табл. 4.

Для оценки качества модели дополнительно рассчитывается средняя относительная погрешность оценки и среднеквадратическая ошибка оценки.

Средняя относительная погрешность оценки определяется по формуле (7):

$$
\Delta=\frac{100 \%}{n} \cdot \sum \frac{\left|Y_{l}-Y_{i}\right|}{Y_{l}},
$$

где $Y_{l}$ - реальное значение рыночной стоимости; $Y_{i}$ - модельное значение рыночной стоимости; $n$ объем выборки. 
Таблица 4. Проверка статистической значимости модели для двух вариантов

Table 4. Checking statistical importance of the model for two options

\begin{tabular}{|c|c|c|c|c|c|}
\hline \multirow[b]{2}{*}{ 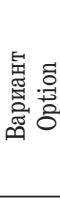 } & \multirow[b]{2}{*}{$\begin{array}{l}\text { Модель } \\
\text { Model }\end{array}$} & \multirow{2}{*}{ 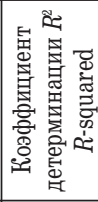 } & \multicolumn{2}{|c|}{$\begin{array}{c}\text { Критерий Фишера } \\
\text { F-test } \\
\end{array}$} & \multirow{2}{*}{$\begin{array}{c}\text { Статистичес- } \\
\text { кая значи- } \\
\text { мость модели } \\
\text { Statistical sig } \\
\text { nificance of } \\
\text { the model }\end{array}$} \\
\hline & & & $\begin{array}{c}\text { расчет- } \\
\text { ный } \\
\text { calculated }\end{array}$ & $\begin{array}{c}\text { таблич- } \\
\text { ный } \\
\text { standard }\end{array}$ & \\
\hline 1 & \multirow{2}{*}{$\begin{array}{c}\text { Регрессион- } \\
\text { ная линейная } \\
\text { Linear } \\
\text { regression }\end{array}$} & 0,73 & 53,23 & 3,86 & \multirow{2}{*}{ да/yes } \\
\hline 3 & & 0,74 & 186,88 & 3,86 & \\
\hline
\end{tabular}

Среднеквадратическая ошибка оценки (стандартное отклонение), характеризующая разброс модельных оценок относительно существующих значений рыночных цен $1 \mathrm{~m}^{2}$, рассчитана по формуле (8):

$$
\sigma=\sqrt{\frac{\sum\left(Y_{l}-Y_{i}\right)^{2}}{N-m-1}},
$$

где $N$ - объем выборки; $m$ - количество факторов стоимости.

Критериями отбора модели являются следующие показатели:

- минимальная средняя относительная погрешность;

- максимальный коэффициент детерминации;

- минимальная среднеквадратическая ошибка.

По показателям «средняя относительная погрешность оценки», «Коэффициент детерминации», «среднеквадратическая ошибка оценки» обе модели признаны статистически значимыми.

После выбора модели расчета кадастровой стоимости необходимо экстраполировать ее на все оцениваемые земельные участки. Модельная рыночная стоимость, получаемая в результате расчета, будет являться удельными показателями кадастровой стоимости оцениваемых земельных участков.

Кадастровая стоимость определяется путем перемножения удельного показателя кадастровой стоимости на площадь земельного участка.

Итоговые значения кадастровых стоимостей земельных участков для двух вариантов учета факторов показали, что разница средних значений полученных кадастровых стоимостей составляет $15 \%$. Этот факт подтверждает гипотезу о том, что от выбора метода учета ценообразующих факторов зависят итоговые значения кадастровых стоимостей.

\section{Обсуждение}

В работе затронута актуальная проблема одного из этапов проведения государственной кадастровой оценки земель населенных пунктов - отбор и учет наиболее значимых ценообразующих факторов.

В рамках исследования проведен анализ зависимости кадастровой стоимости земель населенных пунктов от ценообразующих факторов, влия- ние каждого из которых было выражено через систему разноразмерных оценочных показателей. В результате выявлены основные факторы, оказывающие влияние на величину кадастровой стоимости земель, а именно: площадь участка, доступность учреждений культуры, объектов индустрии отдыха и развлечений, организаций сферы обслуживания, высших учебных и средних специальных учебных заведений, уровень развития транспортной инфраструктуры. Доказана возможность вариативного учета влияния факторов на стоимость земель с помощью системы разноразмерных оценочных показателей.

Такой инструмент управления, как оценка земель, должен рассматриваться как система, способная адаптироваться к постоянным изменениям состояния качества окружающей среды, степени развития инженерной и производственной инфраструктуры, социально-бытового обслуживания населения, состояния инженерно-геологических условий и подверженности территории разрушительным природным и техногенным воздействиям. Одним из вариантов реализации быстрого учета вышеописанных изменений, информация о которых необходима при проведении кадастровой оценки, является создание баз данных, в которых в том числе будет содержаться постоянно обновляемая информация о факторах и показателях кадастровой стоимости для каждого отдельного района города. Создание подобной базы данных на этапе разработки потребует определенного количества затрат труда и времени, однако в дальнейшем позволит ускорить процесс и повысить качество кадастровой оценки недвижимости. Данный метод способен повысить точность результатов оценки объектов недвижимости в долгосрочной перспективе.

В заключение нужно отметить, что для повышения точности результатов кадастровой оценки необходимо совершенствование существующих методов кадастровой оценки недвижимости. Результаты данного исследования могут быть использованы при разработке методического обеспечения массовой кадастровой оценки земель населенных пунктов России.

\section{Заключение}

В настоящей статье проведено исследование влияния варианта учета ценообразующих факторов социальной инфраструктуры на примере земельных участков Василеостровского района города Санкт-Петербург.

По результатам проведенного исследования можно сделать следующие выводы:

1. При проведении государственной кадастровой оценки земель населенных пунктов необходимо учитывать существование вариантов учета ценообразующих факторов. Вариативный учет факторов при оценке одного и того же объекта недвижимости приводит к получению различных величин кадастровой стоимости объектов, 
поэтому выбор того или иного варианта учета факторов должен быть обоснован.

2. Корреляционный анализ оценочных показателей факторов выявил различную степень их зависимости с рыночными стоимостями объектов недвижимости, что говорит о том, что они не могут быть взаимозаменяемы при построении статистических моделей кадастровой стоимости.

\section{СПИСОК ЛИТЕРАТУРЫ}

1. Чернецкая Ю.В. Кадастровая оценка земельных участков индивидуальной жилой застройки населенных пунктов с учетом обременений и ограничений: автореф. дис. ... канд. техн. наук. - СПб, 2014. - 20 c.

2. Шабаева Ю.И. Кадастровая оценка земель индивидуальной жилой застройки с учетом дифференциации городской территории по престижности: автореф. дис. ... канд. техн. наук. СПб, 2015. - 20 с.

3. Трибуц 0.А. Кадастровая оценка земель жилой застройки с учетом влияния разломов: автореф. дис ... канд. техн. наук. СПб, 2012. - 20 с.

4. Шабаева Ю.И. Групповая экспертная оценка значимости факторов на основе использования метода парного сравнения // Инженерный вестник Дона. - 2014. - № 4. - С. 47-53.

5. Faris AL-Oqla D.D., Hayajneh M. Application of the Analytic Hierarchy Process (AHP) in MultiCriteria Analysis of the Selection of Cranes / Jordan Journal of Mechanical and Industrial Engineering. - 2010. - № 5. - P. 567-578.

6. Балтыжакова Т.И. Кадастровая оценка земель малых и средних населенных пунктов с учетом взаимной зависимости ценообразующих факторов: автореф. дис. ... канд. техн. наук. СПб, 2015. - 20 c.

7. Балтыжакова Т.И. Выявление скрытых факторов кадастровой оценки земель населенных пунктов методом главных компонент // Инженерный вестник Дона. - 2015. - № 1-1. - С. 20-25.

8. Демидова П.М., Рыбкина А.М. Детерминистические методы интерполяции и возможность их применения для целей кадастровой оценки земель малоэтажной жилой застройки населенных пунктов // Международный научно-исследовательских журнал. - 2016. - № 11-5 (53). - С.70-72.

9. Чирухин А.В. Современные требования к научно-экономическому обоснованию кадастровой оценки // Научное обеспечение реализации государственных программ АПК и сельских территорий: Материалы международной научно-практической конференции. - Курган: Курганская ГСХА, 2017. C. $161-164$.

10. Рубинштейн Е.Д., Осипенко Е.Д. Анализ рынка недвижимости и его прогнозирование. Теория и практика общественного развития. - 2015. - № 12. - С. 140-143.

11. Baumane V. Cadastral Valuation Models // Economic Science for Rural Development: proceedings of international scientific conference. - Craiova, Romania, 2010. - № 22. - P. 68-75.
3. В продолжение исследований авторов следует отметить целесообразность создания классификатора ценообразующих факторов, в котором были бы типизированы объекты оценочных показателей для каждого фактора и в первую очередь учитывались бы объекты, имеющие набольшее предпочтение у потенциальных покупателей недвижимости.

12. Baumane V. Evaluation of indicators of cadastral assessment // Civil Engineering 13: Proc. $4^{\text {th }}$ International Conference. P. I. Land Management and Geodesy. - Jelgava, Latvia University of Agriculture, 2013. - P. 299-304.

13. Bagdonavicius A., Deveikis S. Individual and Mass Valuation Present and Future // Pharaohs to Geoinformatics: Proc. of FIG Working Week 2005 and GSDI-8. - Cairo, Egypt, April 16-21, 2005. - P. 1-5.

14. Baumane V. Content and application possibilities of evaluation methods in real property cadastral assessment in Latvia // Baltic Surveying'11. Proc. of International Scientific Conference. - Jelgava, LUA, 2011. - P. 43-50.

15. Горшков С.И. Оспаривание результатов кадастровой оценки объектов недвижимости // Научные труды Северо-западного института управления. - 2016. - № 3 (20). - Т. 6. - С. 246-254.

16. Лыжко Е.А. Кадастровая оценка объектов недвижимости и процедура ее оспаривания // Инновационная деятельность: теория и практика. -2016 . - № 7 (3). - С. 3-7.

17. Лепихина 0.Ю., Гореликов В.Г. Проблемы кадастровой оценки земель жилой застройки малых и средних городов России // Записки Горного института. - 2013. № 210. - С. 175-181.

18. Якупова Н.М., Галимова Л.И. Проблемы оценки кадастровой стоимости земельных участков // Фундаментальные исследования. - 2016. - № 7 (Ч. 2). - С. 417-422.

19. Ковязин В.Ф., Романчиков А.Ю. Проблемы кадастровой оценки лесных земель с учетом инфраструктуры лесного фонда // Записки Горного института. - 2018. - № 229. - С. 98-104.

20. Adrian Savu, Daniela Raboj. Cadastral Documentation in Real Valuation // The Valuation Journal. - 2014. - V. 9. - Iss. 1. P. 54-71.

21. Giluca A., Parsova V., Zgirskis M. Use of analytic hierarchy process in evaluation of land survey projects case in Latvia // Baltic Surveying'11. Proc. of International Scientific Conference. - Jelgava, LUA, 2011. - P. 78-83.

22. Pravdina E.A., Lepikhina 0.J. Influence of pricing factor dimension on the cadastral cost of urban land plot // Journal of environmental management and tourism. - 2016. - V. 7. - Iss. 3 (15). P. 383-393.

Поступила 11.05.2018 2.

\section{Информация об авторах}

Лепихина О.Ю., кандидат технических наук, доцент кафедры инженерной геодезии Санкт-Петербургского горного университета.

Правдина E.A., кандидат технических наук, доцент кафедры маркшейдерского дела Санкт-Петербургского горного университета. 
UDC 519.23

\section{VARIABLE ACCOUNTING OF PRICING FACTORS AT LAND PARCELS CADASTRAL VALUATION (ON THE EXAMPLE OF SAINT-PETERSBURG)}

Olga Yu. Lepikhina',

Olgalepikhina1984@gmail.com

Ekaterina A. Pravdina',

eanes@yandex.ru

'Saint-Petersburg Mining University,
2, 21st str. V.O., Saint-Petersburg, 199026, Russia.

The relevance. The earth is the most valuable resource, the importance of which is now very difficult to overestimate. Being a means of production, at the same time, it is as a spatial basis for population activity. In today's environment, the issue of ensuring rational and efficient use of land is particularly acute. For this purpose complex analysis and estimation of its economic, climatic, ecological, geological and other characteristics is necessary. One of the types of such assessment is definition of cadastral value of land. In our country it is used mainly for taxation, therefore, it is important to provide accurate and objective results of the assessment. Modern techniques are not perfect, and their use does not always provide the correct and fair value of land. For example, not enough attention is paid for choosing source factors and justification of variant of their accounting at cadastral valuation. Each of these factors may be accounted by some indicators with different dimensions. The article is devoted to the analysis of dependence between chosen indicators and cadastral value on the example of land parcels which are located on the Vasilievsky Island in Saint-Petersburg.

The main aim of research is to study the influence of variable accounting of pricing factors on the total cost of urban lands for further specification and improvement of land parcels cadastral valuation methods.

Methods. The analysis of objects location was carried out by studying the cartographical sources as well as by visual survey of real estate objects. The values of estimated indicators of land parcels were gathered by the methods of spatial analysis and modeling in geographic information system Maplnfo. The land parcels market data were selected by the methods of statistical collecting and data processing with application of MS Office software and SPSS. The authors used the correlation method and regression analysis for statistical models creation and the method of "correlation galaxies» by P.V. Terentev for defining the interdependent estimated indicators at three considered options.

Results. When carrying out the cadastral valuation of lands it is necessary to consider the existence of variability of factors accounting by some estimated indicators with different dimensions. Choosing this or that indicator characterizing the influence of a factor should be justified properly. The correlation analysis of indicators revealed different degree of their interdependence that demonstrates that they can be not always interchangeable at creation of statistical models. Structure of the most significant indicators such as the calculated cadastral values depends on the choice of structure of initial estimated indicators of factors.

Key words:

Land plot, natural resource, cadastral valuation, pricing factor, estimated indicator, real estate market, correlation and regression method, method of correlation galaxies.

\section{REFERENCES}

1. Chernetskaya Yu.V. Kadastrovaya otsenka zemelnykh uchastkov individualnoy zhiloy zastroyki gorodskikh naselenykh punktov s uchetom obremeneniy i ogranicheniy. Avtoreferat Kand. Nauk [Cadastral assessment of individual housing lands of cities accounting restrictions. Cand. Diss. Abstract]. Saint-Petersburg, 2014. $20 \mathrm{p}$.

2. Shabayeva Yu.I. Kadastrovaya otsenka zemel individualnoy zhiloy zastroyki s uchetom differentsiatsii gorodskoy territorii po prestizhnosti. Avtoreferat Kand. Nauk [Cadastral assessment of individual housing lands of cities accounting urban area zoning by the criteria of the prestige value. Cand. Diss. Abstract]. SaintPetersburg, 2015. $20 \mathrm{p}$.

3. Tributs 0.A. Kadastrovaya otsenka zemel zhiloy zastroyki naselenykh punktov s uchetom viiyaniya razlomov. Avtoreferat Kand. Nauk. [Cadastral assessment of residential development lands of cities accounting influence of breaks. Cand. Diss. Abstract]. Saint-Petersburg, 2012. 20 p.

4. Shabayeva Yu.I. Group expert assessment of the importance of factors on the basis of the pair comparison method. Engineering bulletin of Don, 2014, no. 4, pp. 47-53. In Rus.

5. Faris AL-Oqla D.D., Hayajneh M. Application of the Analytic Hierarchy Process (AHP) in MultiCriteria Analysis of the Selection of Cranes. Jordan Journal of Mechanical and Industrial Engineering, 2010, no. 5, pp. 567-578.
6. Baltyzhakova T.I. Kadastrovaya otsenka zemel malykh i srednikh naselenykh punktov s uchetom vzaimnoy zavisimosti tsenoobrazuyushchikh faktorov. Avtoreferat Kand. nauk [Cadastral assessment of small and average settlement lands accounting dependence of pricing factors. Cand. Diss. Abstract]. Saint-Petersburg, $2015.20 \mathrm{p}$.

7. Baltyzhakova T.I. Identification of the hidden factors of urban lands cadastral value by the method of main components. Engineering bulletin of Don, 2015, no. 1-1, pp. 20-25. In Rus.

8. Demidova P.M., Rybkina A.M. Deterministic methods of interpolation and possibility of their application for cadastral valuation of low residential urban lands. International research journal, 2016, no. 11-5 (53), pp. 70-72. In Rus.

9. Chirukhin A.V. Sovremennye trebovaniya k nauchno-ekonomichskomu obosnovaniyu kadastrovoy otsenki [Modern requirements to scientific and economical justification of cadastral valuation]. Nauchnoe obespechenie realizatsii gosudarstvennykh program APK $i$ selskikh territoriy. Materialy mezhdunarodnoy nauchno-prakticheskoy konferentsii [Scientific support of state programs of farming sector and rural regions. Materials of international scientific conference]. Kurgan, 2017. pp. 161-164.

10. Rubinshteyn E.D., Osipenko N.S. Analiz rynka nedvizhimosti i ego prognozirovanie [The real estate market analysis and forecasting]. Theory and practice of public development, 2015, no. 12, pp. 140-143. 
11. Baumane V. Cadastral Valuation Models. Economic Science for Rural Development: proceedings of international scientific conference, Craiova, Romania, 2010, no. 22, pp. 68-75.

12. Baumane V. Evaluation of indicators of cadastral assessment. Proc. $4^{\text {th }}$ International Conference Civil Engineering'13. P. I. Land Management and Geodesy, Latvia University of Agriculture, 2013. pp. 299-304.

13. Bagdonavicius A., Deveikis S. Individual and Mass Valuation Present and Future. Pharaohs to Geoinformatics. Proc. of FIG Working Week 2005 and GSDI-8. Cairo, Egypt, April 16-21. 2005. pp. 1-5.

14. Baumane V. Content and application possibilities of evaluation methods in real property cadastral assessment in Latvia. Baltic Surveying'11. Proc. of International Scientific Conference. Jelgava, LUA, 2011. pp. 43-50.

15. Gorshkov S.I. Osparivanie rezultatov kadastrovoy otsenki obektov nedvizhimosti [Contesting of real estate cadastral assessment]. Scientific materials of the Northwest institute of management, 2015, vol. 6, no. 3 (20), pp. 246-254.

16. Lyzhko E.A. Cadastral assessment of real estate and its contesting. Innovative activity: theory and practice, 2016, no. 7 (3), pp. 3-7. In Rus.
17. Lepikhina 0.Yu., Gorelikov V.G. Problems of state cadastral assessment of residential lands of small and average towns in RussiaJournal of Mining Institute, 2013, no. 210, pp. 175-181. In Rus.

18. Yakupova N.M., Galimova L.I. Problems of cadastral valuation of land parcels. Fundamental inquires, 2016, no. 7 (P. 2), pp. 417-422. In Rus.

19. Kovyazin V.F., Romanchikov A.Yu. Problems of cadastral assessment of forestland accounting the infrastructure of forest fund. Journal of Mining Institute, 2018, no. 229, pp. 98-104. In Rus.

20. Adrian Savu, Daniela Raboj. Cadastral Documentation in Real Valuation. The Valuation Journal, 2014, vol. 9, Iss. 1, pp. 54-71.

21. Giluca A., Parsova V., Zgirskis M. Use of analytic hierarchy process in evaluation of land survey projects case in Latvia. Baltic Surveying'11. Proc. of International Scientific Conference. Jelgava, LUA, 2011. pp. 78-83.

22. Pravdina E.A., Lepikhina 0.J. Influence of pricing factor dimension on the cadastral cost of urban land plot. Journal of environmental management and tourism, 2016, vol. 7, Iss. 3 (15), pp. 383-393.

Received: 11 May 2018.

\section{Information about the authors}

Olga Yu. Lepikhina, Cand. Sc., associate professor, Saint-Petersburg Mining University.

Ekaterina A. Pravdina, Cand. Sc., associate professor, Saint-Petersburg Mining University. 